\title{
A qualitative synthesis of trials promoting physical activity behaviour change among post-treatment breast cancer survivors
}

\author{
Camille E. Short • Erica L. James • Fiona Stacey • \\ Ronald C. Plotnikoff
}

Received: 12 April 2013 / Accepted: 4 June 2013 / Published online: 26 July 2013

(C) The Author(s) 2013. This article is published with open access at Springerlink.com

\begin{abstract}
Background Health outcome trials have provided strong evidence that participating in regular physical activity can improve the quality of life and health of post-treatment breast cancer survivors. Focus is now needed on how to promote changes in physical activity behaviour among this group. Purpose This systematic review examines the efficacy of behavioural interventions for promoting physical activity among post-treatment breast cancer survivors.

Methods Behavioural intervention studies published up until July 2012 were identified through a systematic search of two databases: MEDLINE and CINAHL, and by searching reference lists of relevant publications and scanning citation libraries of project staff.

Results Eight out of the ten identified studies reported positive intervention effects on aerobic physical activity behaviour,
\end{abstract}

\author{
C. E. Short $(\bowtie)$ \\ Centre for Physical Activity Studies, Institute for Health \\ and Social Science Research, Central Queensland \\ University, Queensland, Australia \\ e-mail: c.short@cqu.edu.au
}

C. E. Short $\cdot$ E. L. James $\cdot$ F. Stacey

School of Medicine and Public Health, Priority Research Centre for

Health Behaviour, University of Newcastle, Callaghan, Australia

\section{E. L. James}

e-mail: Erica.James@newcastle.edu.edu

F. Stacey

e-mail: Fiona.Stacey@newcastle.edu.au

C. E. Short $\cdot$ E. L. James $\cdot$ F. Stacey $・$ R. C. Plotnikoff

Priority Research Centre for Physical Activity and Nutrition,

University of Newcastle, Callaghan, Australia

R. C. Plotnikoff

e-mail: Ron.Plotnikoff@newcastle.edu.au ranging from during the intervention period to 6 months post-intervention. Only two studies reported intervention effect sizes. The identification of factors related to efficacy was not possible because of the limited number and heterogeneity of studies included, as well as the lack of effect sizes reported. Nonetheless, an examination of the eight studies that did yield significant intervention effects suggests that 12 -week interventions employing behaviour change techniques (e.g., selfmonitoring and goal setting) derived from a variety of theories and delivered in a variety of settings (i.e., one-on-one, group or home) can be effective at changing the aerobic physical activity behaviour of breast cancer survivors in the mid- to long terms. Conclusions Behavioural interventions do hold promise for effectively changing physical activity behaviour among breast cancer survivors. However, future research is needed to address the lack of studies exploring long-term intervention effects, mediators of intervention effects and interventions promoting resistance-training activity, and to address issues impacting on validity, such as the limited use of objective physical activity measures and the use of convenience samples.

Implications for Cancer Survivors Identifying effective ways of assisting breast cancer survivors to adopt and maintain physical activity is important for enhancing the well-being and health outcomes of this group.

Keywords Behaviour change interventions - Breast cancer . Randomised controlled trials

\section{Introduction}

Due to earlier detection and advances in treatment, more and more women are surviving breast cancer each year [1]. Whilst improved survival is duly welcomed, breast cancer 
survivors are faced with both short- and long-term health and psychosocial sequelae [2]. To improve the quality of life of breast cancer survivors and negate the associated health burdens and risks, effective health promotion approaches are required [3]. One promising cancer recovery approach is the promotion of regular physical activity.

Evidence from dozens of health outcome trials suggests that regular physical activity can address both the psychological and physiological burdens presented after breast cancer diagnosis and treatment [4-6]. Furthermore, observational research suggests that regular physical activity may also have an impact on survival, with breast cancer survivors who are active after treatment having a lower risk of cancer recurrence, co-morbidities and death from all causes compared with those who are less active, regardless of cancer stage [7-9]. In recognition of these benefits, detailed exercise prescription guidelines for cancer survivors have been published by professional bodies internationally [10-14]. Despite these recommendations, the majority of breast cancer survivors are not sufficiently active to obtain health benefits [15-18] and efforts to encourage regular physical activity are not a routine part of the cancer rehabilitation process in most centres [19-22].

Given that strong evidence now exists supporting the safety and efficacy of physical activity interventions for enhancing health outcomes $[6,23,24]$, there is a need to direct attention to physical activity promotion strategies that can be delivered to survivors in an effective and sustainable way. Whilst supervised exercise programs of short duration were highly appropriate for establishing safety and assessing the impact of regular physical activity on health outcomes (such as fatigue and quality of life), interventions that promote sustainable behaviour change are now required. To increase the likelihood of success, interventions should be based on empirical research evidence and grounded in strong health behavioural theory $[25,26]$. To inform this process, a detailed synthesis of the physical activity behaviour change literature specific to breast cancer survivors is required. Two comprehensive reviews related to this topic have already been conducted $[6$, 27]. However, neither provides a synthesis of studies exploring the efficacy of behavioural intervention strategies for promoting the adoption and maintenance of physical activity among those in the post-treatment phase. Rather, White et al. [6] examined the potential for physical activity interventions to be translated into practice. Many of the studies included in the White et al. review were highly controlled health outcome trials, designed to demonstrate the impact of regular physical activity on health outcomes, as opposed to behaviour change trials designed to promote changes in physical activity behaviour. Whilst it is becoming a common practice to include behavioural support in physical activity interventions, evaluations of such trials provides very little insight into how to promote sustainable behaviour change if descriptions of behavioural strategies and behavioural outcomes are not reported or adequately described [28]. As such, it is not surprising that White et al. concluded that many of the studies lacked external validity, hindering the translation of the interventions into practice. The review conducted by Spark et al. [27] did focus on behavioural interventions, but only maintenance of intervention effects were explored (studies not reporting post-intervention follow-ups were excluded) and furthermore, the included studies were not restricted to posttreatment breast cancer survivors (i.e. studies conducted on mixed cancer samples and breast cancer patients undergoing treatment were included in the review). Whilst physical activity is a recommended cancer control strategy across all cancerrelated time periods (i.e. pre-diagnosis to-end of life), physical activity for recovery, rehabilitation and health promotion is recommended among survivors in the period after treatment and prior to the development of a recurrence of cancer or death [29]. Therefore, it is important to review the specific literature on the efficacy of behavioural interventions on both the adoption and maintenance of physical activity among post-treatment breast cancer survivors $[11,29]$. The primary purpose of this review is to synthesise this literature and provide direction for future research. Given the known heterogeneity of behaviour change trials and the small number of studies conducted in this field, a qualitative synthesis of the literature is provided in this systematic review.

\section{Method}

\section{Eligibility criteria}

Studies were eligible for inclusion in this review if they: (a) examined the efficacy of at least one behaviour modification intervention designed to promote physical activity (i.e. aerobic activity and/or resistance training) among adult posttreatment (not including hormone therapy) breast-cancer survivors; (b) if they included either self-reported or objectively assessed physical activity behaviour change as a study outcome; and (c) used an individual or cluster randomised controlled design. Interventions were considered to be a 'behaviour modification intervention' if they included at least one behaviour change strategy (e.g. goal-setting, selfmonitoring, problem solving and health education; see Michie et al. [29] for taxonomy of behaviour change techniques relating to changing physical activity and healthy eating behaviours) aimed at promoting enhanced physical activity behaviour [29]. Studies testing the efficacy of interventions targeting multiple behaviours (including physical activity) were included.

Studies were excluded if they: (a) were published in a language other than English, (b) reported the efficacy of a physical activity intervention that did not involve behaviour 
change techniques (for example, a supervised exercise program with no intervention component targeting increased knowledge or skills), (c) included mixed samples of cancer survivors (including breast cancer survivors) and did not report intervention effects specifically by cancer type, (d) included breast cancer survivors still undergoing active treatment (defined as: surgery, chemotherapy and radiotherapy) or (e) were available as a conference abstract only.

Initially, articles were assessed for eligibility by a single reviewer based on the study title and abstract. After this initial screening, one third of full-text articles (selected alphabetically in ascending order) were assessed for eligibility independently by two reviewers using a purpose-designed screening tool (applying inclusion criteria hierarchically). Findings were compared and disagreements between reviewers were resolved by consensus. The remaining two thirds were screened by one reviewer using the screening tool.

\section{Search strategy}

Studies were identified through a structured electronic database search of all publication years (until July 2012) in MEDLINE, and CINAHL. The following search terms were used: exercise (or physical activity or motor activity) and cancer (or neoplasms) and randomised controlled trial(s) (or controlled clinical trial, intervention studies or clinical trial). Results were limited to articles published in the English language and studies conducted among humans. Reference lists of relevant articles, identified reviews and files of project staff were also scanned to check for studies not identified via the electronic search process.

\section{Data extraction}

The abstraction form for the Guide to Community Preventive Services [30] was used as a template for article abstraction. This tool includes questions about study design and execution, number and characteristics of participants, participant recruitment, and details of the intervention (such as theoretical underpinning, dose of physical activity and non-physical activity components). Tables of study descriptions and outcomes were developed and reviewed by a second study investigator for completeness and accuracy.

Follow-up periods were divided into three categories: short ( $<3$ months post-intervention), medium (3-6 months post-intervention) and long terms ( $>6$ months post-intervention). Key methodological quality of each study was assessed independently by two reviewers using the McMaster quality assessment tool for quantitative studies developed by the Effective Public Health Practice, Canada [31]. Findings were compared and disagreements between reviewers were resolved by consensus.

\section{Results}

Study selection

The initial search of the electronic database yielded 1,397 publications, which were reduced to 347 following the review of study titles and abstracts. After removing duplicates and reviewing full-text articles, nine trials [32-49] (published in 17 articles) met the eligibility criteria. Checking project staff files identified one additional paper [50]. Reference checking did not yield any additional papers (see Fig. 1 for PRISMA diagram summarising the selection process).

Trial characteristics

An overview of the studies included is provided in Table 1. Five of the ten included studies specified physical activity behaviour change as a primary outcome [37, 39-41, 50]. Primary outcomes in the remaining studies were physical performance [33], quality of life [35], feasibility [36] and weight loss [38]. Most studies focused on promoting regular participation in moderate-vigorous aerobic physical activity and only one study focused on promoting resistance training [34]. Seven studies focused on the promotion of physical activity alone [33, 35, 37, 39-41, 50], whilst the remainder promoted physical activity and at least one other behaviour (e.g. diet) [34, 36, 38]. Self-report measures were used in all studies, but four studies also included an objective measure of aerobic activity (three utilising accelerometers [37, 39, 40] and one utilising pedometers [41]). Trial participants were predominately recruited via advertisements, and invitations sent via cancer care centres or treating oncologists/surgeons. Two studies recruited participants via cancer registry [34, 41]. Eligibility criteria differed between studies in regards to time since active treatment and cancer stage. Three studies targeted survivors who had recently completed treatment [35-37] (ranging from treatment completed within the last year to 3 years); three studies restricted participation to those within a certain number of years from diagnosis (i.e. within 5 years from diagnosis [39], within 7 years from diagnosis [33] and within 14 years from diagnosis [38]), and one study focused on survivors who were at the five-year survival mark [34]. The remaining studies did not restrict participation based on time since active treatment $[40,41,50]$. Four studies excluded breast cancer survivors diagnosed with stage 0 breast cancer [37, 38, 40, 41], two studies included these survivors [36, 39], and four studies did not specify if stage 0 breast cancer survivors were included [33-35, 50]. Seven [33, 35-37, 39, 40, 50] of the ten studies identified excluded participants that were already active (although the criteria for 'active' differed between studies). With few exceptions [34, 41], study samples (N's ranged between 36 and 404) were generally small and mainly consisted of middleaged, overweight participants. 
Fig. 1 PRISMA flow diagram summarising selection process

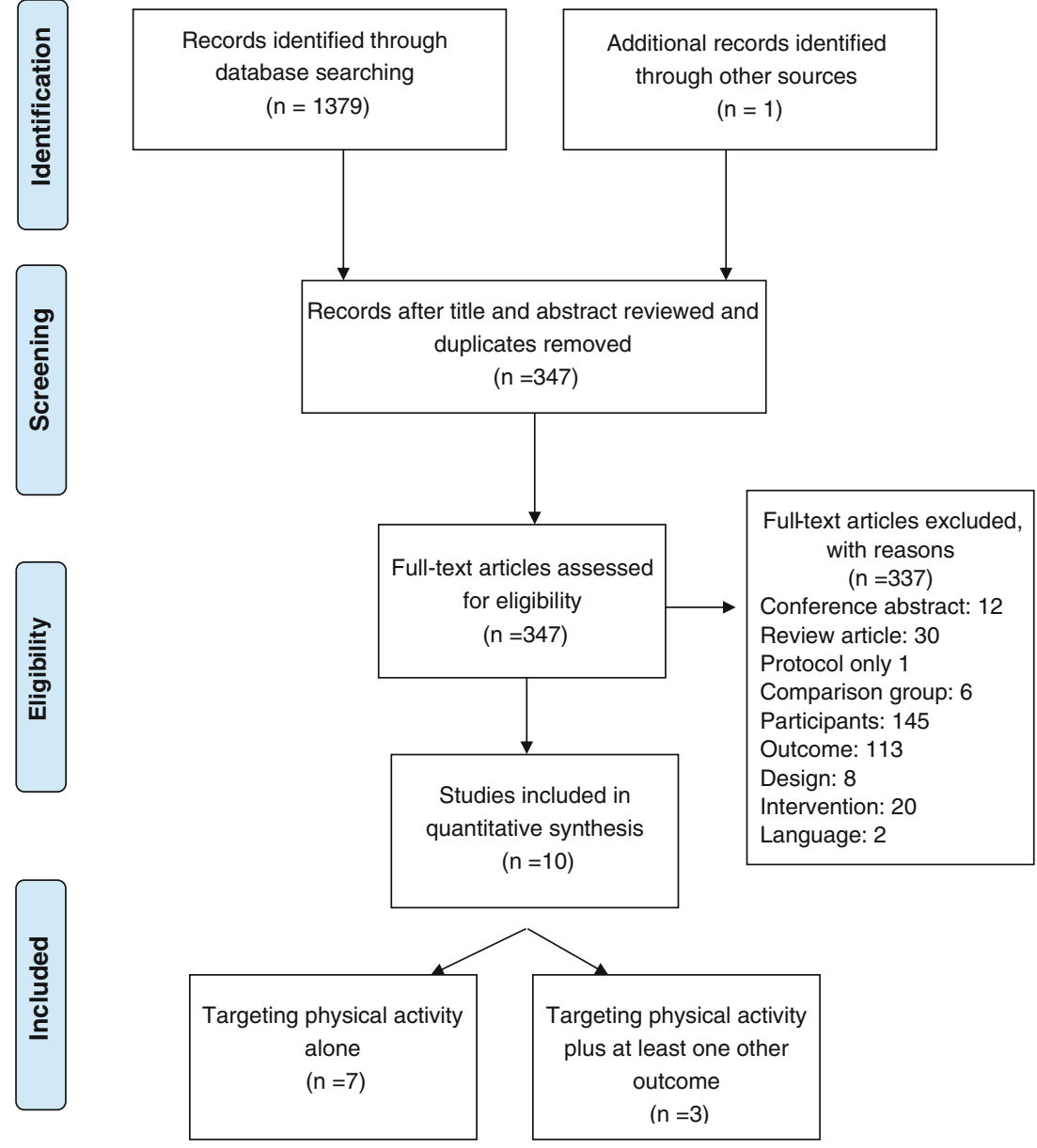

was operationalised in four interventions [33, 35, 36, 39], Social Cognitive Theory [52] was operationalised in three interventions $[37,39,50]$ and the Theory of Planned behaviour [53] was operationalised in one intervention [41]. Of the two remaining interventions, one was guided by a theoretical construct (i.e. social support) [34] and one was atheoretical [38].

\section{Behaviour change techniques}

The most commonly employed behaviour change techniques were self-monitoring and goal-setting [33, 35, 37, 39, 41, 50], eliciting social support $[34,35,37,40,50]$ and positive reinforcement [33, 36, 39, 41]. Time management [40, 50], providing instruction $[34,41]$, cognitive reappraisal and consciousness raising $[35,36]$ and positive role models [40] were also used in some studies.

\section{Comparison groups}

Five studies compared the efficacy of the intervention to a no intervention control [34, 36-38, 50], four studies compared the efficacy of the behaviour change intervention to a standard/usual care condition [33, 35, 40, 41], and one study
Eight of the ten interventions were fully informed by a behaviour change theory and employed techniques related to the theory used. The Transtheoretical Model [51] 


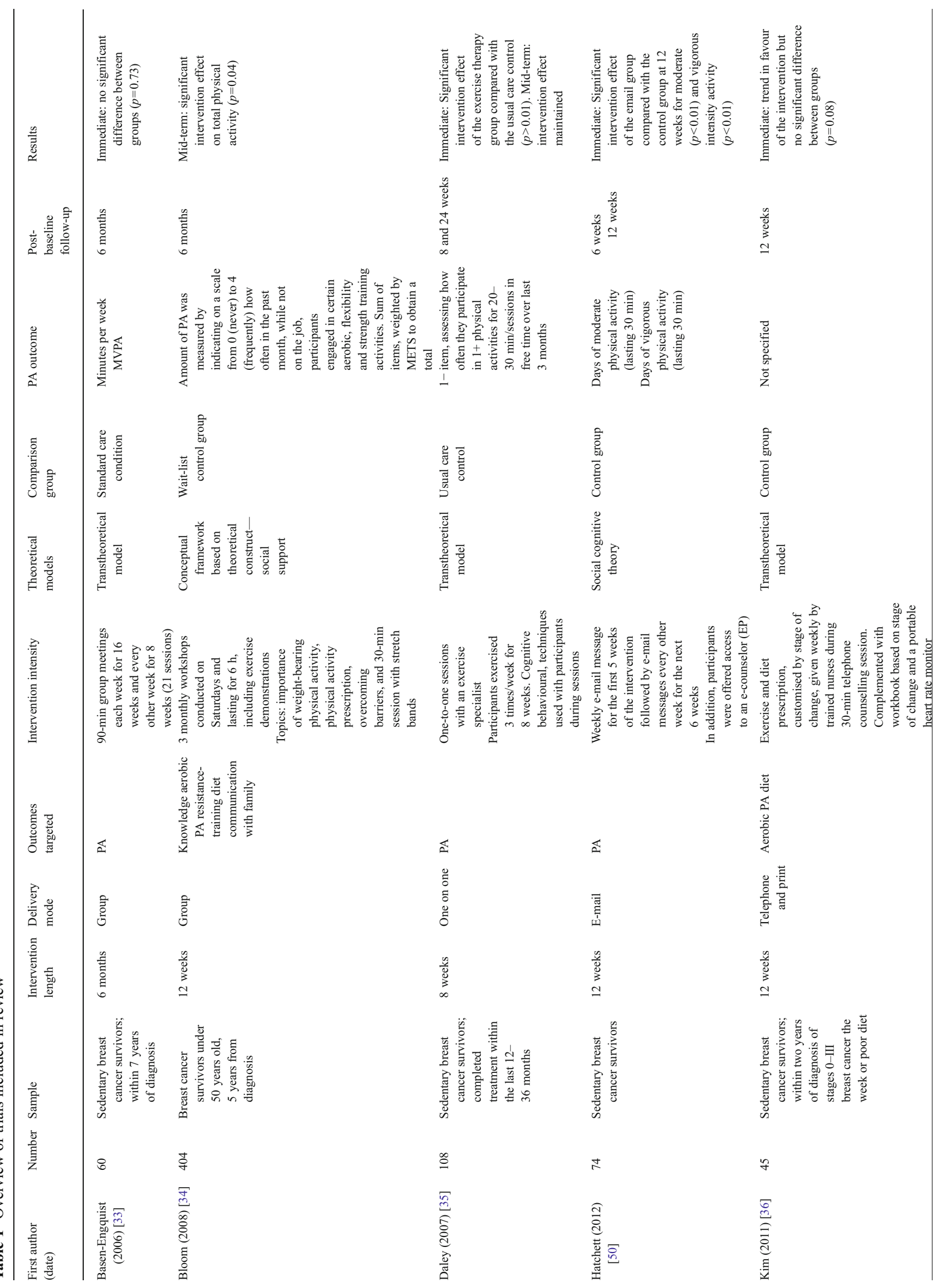




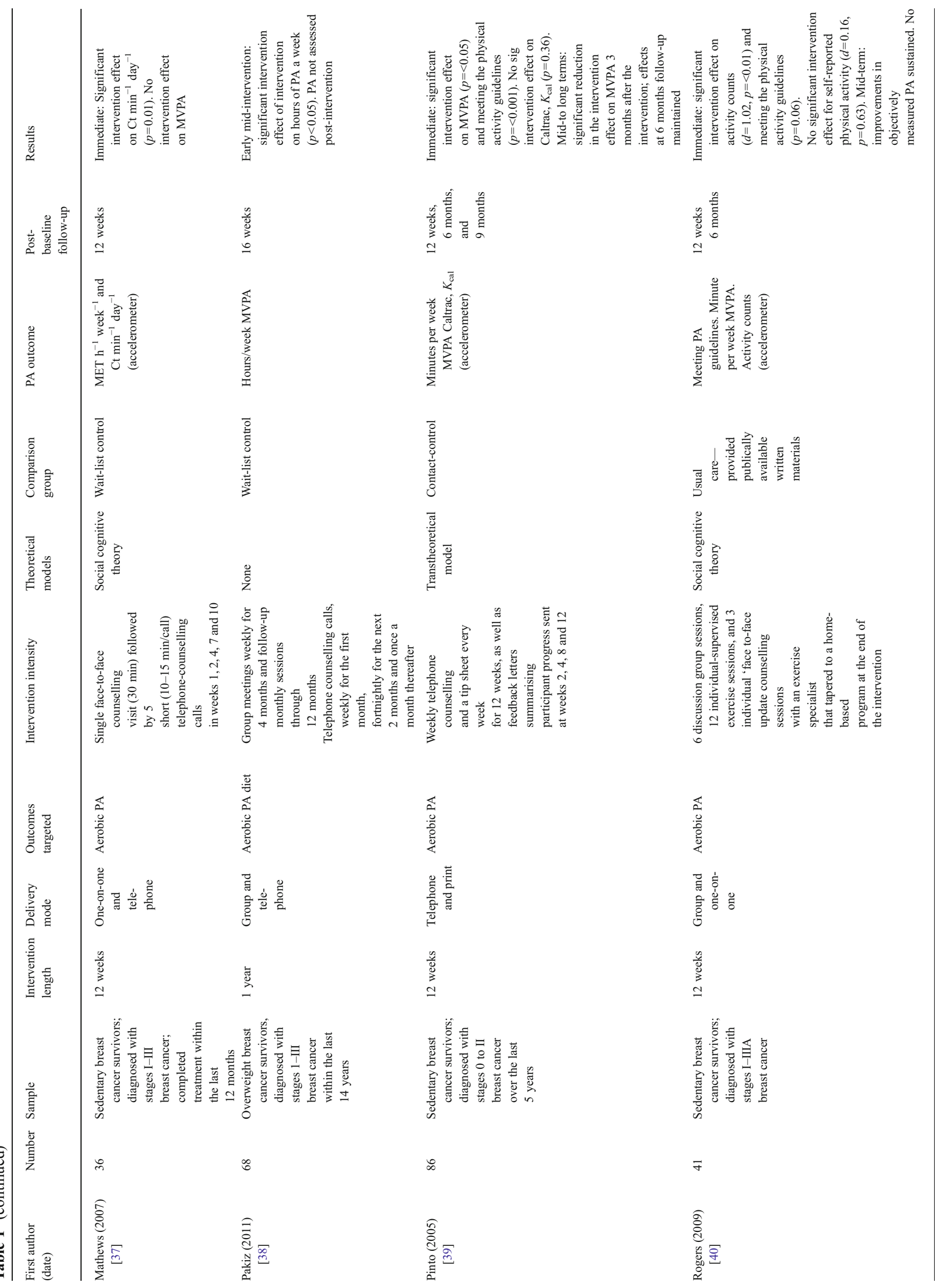




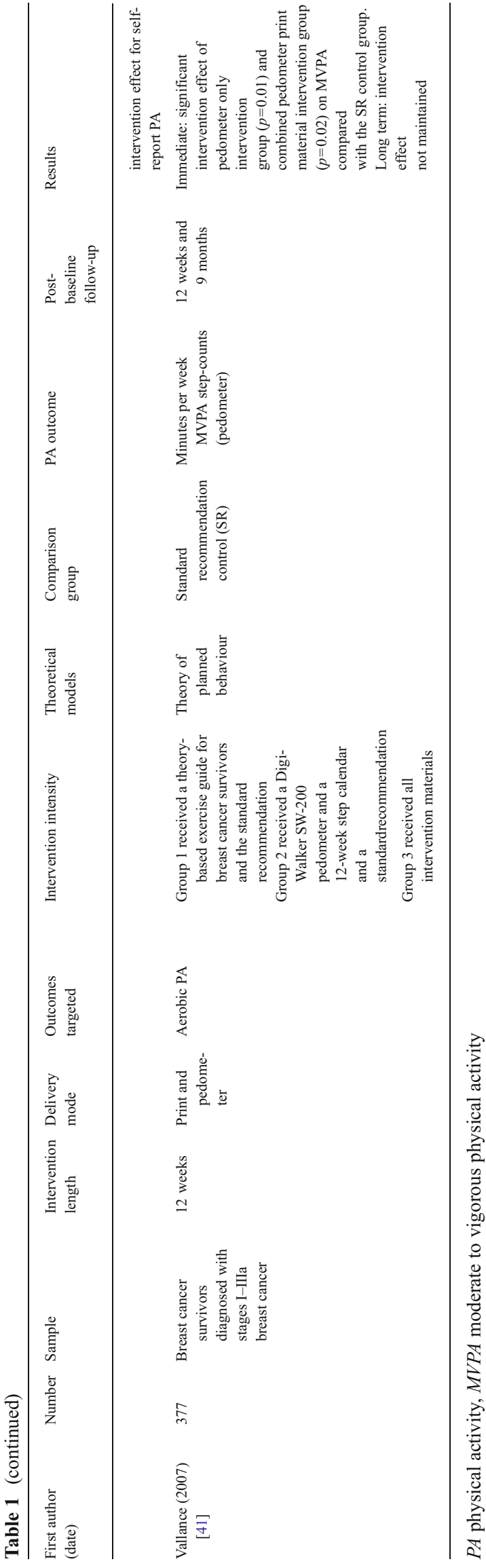

compared the efficacy of the intervention to a contact control condition [39].

Methodological quality

Findings from the methodological review are presented in Table 2. Based on the assessments by two reviewers (CS and FS) using a standardised tool [32], two of the studies were rated as 'strong' [39, 41], five of the studies were rated as 'moderate' $[33,36,38,40,50]$ and three were rated as 'weak' $[34,35,37]$. Issues relating to selection bias (due to low consent rate and/or recruitment method) were considered a methodological limitation in eight studies, failure to report withdrawal and dropout information was an issue in one study, and the reliability and validity of the measurement tool for assessing physical activity was considered an issue in two studies.

Intervention effects on self-report and objectively assessed physical activity

\section{Self-reported physical activity}

Significant intervention effects on self-reported physical activity behaviour change were reported in eight $[34,35$, $37-41,50]$ out of the ten included trials, ranging from halfway through a 12-week intervention [38] to a 9-month follow-up [39] (see Table 1). Of the five studies [34, 35, 39-41] that assessed self-reported behaviour change at a post-intervention follow-up, three reported maintenance of the intervention effect (two mid- [34, 35] and one long terms $[39,42]$ ), whilst two reported that the intervention effect was not sustained (mid- [40] and long terms [41]). Of the two studies that did not find a significant intervention effect on physical activity behaviour change $[33,36]$, one study found a positive intervention trend at post-intervention $(p=0.086)$ and both reported positive changes in motivational readiness for exercise, as well as significant positive changes in other outcomes targeted (i.e. physical performance and bodily pain [33]; diet, emotional functioning, fatigue, and depression [36]). Both of these studies were pilot studies with small sample sizes $(n=60$ [33]; $n=45$ [36]) and may have been underpowered.

\section{Objective measures}

Significant intervention effects on objectively assessed physical activity behaviour change were reported in two [37, 40] out of the four studies that utilised an objective measure, ranging from the short midterm. Interestingly, there was incongruence between the objective and self-report measures employed in all four studies. Namely, those that found significant intervention effects on objectively assessed physical 
Table 2 Methodological quality of the included studies rated by two reviews

\begin{tabular}{|c|c|c|c|c|c|c|c|}
\hline First author (date) & Selection bias & Study design & Confounders & Blinding & $\begin{array}{l}\text { PA outcome } \\
\text { assessment }\end{array}$ & $\begin{array}{l}\text { Withdrawals } \\
\text { and drop outs }\end{array}$ & $\begin{array}{l}\text { Global } \\
\text { rating }\end{array}$ \\
\hline Basen-Engquist (2006) [33] & Weak & Strong & Strong & - & Strong & Strong & Moderate \\
\hline Pinto (2005) [39] & Moderate & Strong & Strong & - & Strong & Strong & Strong \\
\hline Rogers (2009) [40] & Weak & Strong & Strong & - & Strong & Strong & Moderate \\
\hline Mathews (2007) [37] & Weak & Strong & Strong & - & Strong & Weak & Weak \\
\hline Vallance (2007 [41]) & Strong & Strong & Strong & - & Strong & Strong & Strong \\
\hline Hatchett (2012) [50] & Weak & Strong & Strong & - & Strong & Strong & Moderate \\
\hline Daley (2007) [35] & Weak & Strong & Strong & - & Weak & Strong & Weak \\
\hline Kim (2011) [36] & Weak & Strong & Strong & - & Strong & Strong & Moderate \\
\hline Bloom (2008) [34] & Weak & Strong & Strong & - & Weak & Strong & Weak \\
\hline Pakiz (2011) [38] & Weak & Strong & Strong & - & Strong & Strong & Moderate \\
\hline
\end{tabular}

activity (using accelerometers in both cases) [37, 40] did not report significant intervention effects on self-reported physical activity. Whereas, those that did not report significant intervention effects for objectively assessed (via pedometers [41]; or accelerometers [39]) physical activity, did report intervention effects for moderate-vigorous self-reported physical activity.

\section{Effect sizes}

Only two studies reported intervention effect sizes. At the immediate post-intervention follow-up, effect sizes ranged from small to large, and were highest for objectively measured physical activity (large effect size reported for accelerometer assessed physical activity, $d=1.02$, [40]; medium effect size reported for pedometer assessed physical activity, $d=0.62$ [39]; small-medium effect sizes reported for selfreported physical activity, $d=0.16[40] ; d=38[41]$ ). Effect sizes were not reported for post-intervention follow-ups in either of these studies.

\section{Evaluation of factors impacting on intervention efficacy}

The ability to identify and evaluate factors relating to intervention efficacy is limited due to the relatively small number of studies conducted, the heterogeneity of the studies included in terms of the interventions delivered and methodological characteristics, and the absence of effect sizes measures in all but two studies. Nonetheless, an examination of the eight studies that did yield significant intervention effects suggests that 12 week interventions employing behaviour change techniques (e.g., self-monitoring, encouraging social support, goalsetting, providing instruction) derived from a variety of theories and delivered in a variety of settings (i.e., one-on-one, group, home) can be effective at changing the physical activity behaviour of breast cancer survivors immediately after the intervention, with some evidence of maintenance in the mid- to long terms. Furthermore, as there were few differences between the two studies that did not achieve significant changes in physical activity versus those that did, it may be likely that differences in effect were due to power issues rather than differences in intervention characteristics, especially in the trial that found a positive trend $(n=45)[36]$.

\section{Mediators of intervention effects}

Only three studies conducted mediation analyses [47-49] to examine the effect of the intervention on the theoreticallyderived predictors of behaviour change, and to determine if PA was mediated by changes in these variables. Pinto and colleagues [39] found that the positive intervention effect for their home-based intervention, based on the Transtheoretical Model [51], was not mediated by changes in Transtheoretical construct variables [47]. Whereas, positive intervention effects in Rogers and colleagues [40, 48] Social Cognitive Theory-based intervention and Vallance and colleagues [41, 49] Theory of Planned behaviour-based intervention were mediated by changes in the associated theory-based predictors. Namely, in Rogers et al. study [40, 48] barriers interference mediated $39 \%(p=0.004)$ of the intervention effect on PA 3 months post-intervention; and in Vallance et al. study [41, 49, 54] planning and intention were found to partially mediate the effects of the intervention on PA immediately following the 3month intervention, as well as at 3 months post-intervention.

\section{Discussion}

The primary purpose of this review was to synthesise the existing literature relating to the efficacy of behaviour change interventions for promoting physical activity among post-treatment breast cancer survivors and to provide direction for future research. The review shows that while very few trials have been conducted in this domain, behavioural 
interventions of various intensities and delivery modes do hold promise for effectively changing aerobic physical activity behaviour among breast cancer survivors. Of interest to public health researchers and practitioners, one can envisage that several of the efficacious interventions described here could potentially be disseminated in a sustainable way to the increasingly growing population of breast cancer survivors [55]. However, there are gaps in the research to date that need to be addressed to fully inform public health approaches in this area.

First, empirical evidence regarding the ability of these interventions to be adopted, implemented and maintained at an individual and community level is required $[55,56]$. Our findings mirror those of Spark and colleagues [27] who concluded that the majority of studies targeting physical activity among breast cancer survivors do not report maintenance outcomes of the intervention, and few report sustained intervention effects. Furthermore, the majority of studies do not include objective measures of behaviour change. Our findings also mirror those of White and colleagues [6] who concluded that studies targeting physical activity amongst breast cancer survivors often report outcomes at an individual level (e.g. physical activity outcomes) but not at a setting or organisational level (e.g. adoption, implementation and maintenance of intervention by study staff and key stakeholders). This lack of information at both an individual and settings level hinders the validity of the interventions and must be addressed to progress the translation of research into practice. A well-regarded framework for evaluating the external validity and public health impact of health promotion interventions is the RE-AIM framework [57]. The framework consists of five dimensions (i.e., reach, efficacy, adoption, implementation, and maintenance) that occur at multiple levels (e.g., individuals, organisation, and community) and interact to determine the public health impact of the intervention. The guiding premise is that failure to adequately evaluate programs on all five dimensions can lead to a waste of resources, discontinuities between stages of research, and failure to improve public health [55-57]. This would suggest that one direction for future research is to evaluate these interventions at the organisational or community level, using objective measures where possible.

Second, whilst the majority of studies included in this review were theory-based, only three tested the mediating role of the theoretical constructs (targeted by the intervention) on physical activity behaviour. Such analyses are needed to provide insight into 'why' interventions work, and hence accelerate the identification of effective behaviour change techniques and the development of evidence-based practice in the field applied [58]. Interestingly, Spark et al. [27] reported that interventions relying less heavily on a theoretical model for intervention development and reporting the use of fewer behavioural intervention strategies seemed to be more likely to achieve successful maintenance of intervention effects. This was not observed in the current review, and furthermore is in direct contrast to current wisdom regarding the necessity of using theory in the development and evaluation of behavioural interventions $[25,26,30,59]$ and a growing body of evidence suggesting that theory-based interventions are more effective than a-theoretical interventions [60]. As such, we concur with the authors that this finding should be interpreted with caution. One possible explanation is that the results were skewed by the inclusion of health outcome trials that have a behavioural component. Such trials tend to be less reliant on theory than traditional behaviour change trials but in turn are more highly controlled and highly resourced, which may promote greater maintenance of intervention effects [28]. Hence, it is recommended that the development and evaluation of future interventions continue to be based on sound behavioural theory. Without such understanding, the development of effective interventions targeted at this population is likely to be hindered by 'wheels' being reinvented rather than re-applied [58].

Third, information regarding the efficacy of computermediated physical activity interventions in this population group requires further investigation [61]. Only one study included in this review evaluated the efficacy of a computermediated intervention and no mid- or long-term follow-up occurred [50]. Computer-mediated interventions have shown promise in other population groups [61, 62] and may be particularly translatable in chronic disease setting [63], where several stakeholders already have a strong online presence (e.g. The American Breast Cancer Foundation, The Breast Cancer Network, and The Cancer Council). In line with this, a review of chronic illness management interventions using the REAIM framework [55] concluded that whilst traditional faceto-face intervention modalities are often efficacious they may have limited impact if they cannot be delivered consistently to large segments of the target population. Whereas, interventions using new information technologies may have greater reach, adoption, implementation and maintenance, and thereby greater public health impact. Exploring this research avenue further in the breast cancer population may enhance our ability to provide cost effective and sustainable supportive cancer care.

Fourth, only one of the trials included in this review focused on promoting behavioural changes in resistancetraining and the efficacy of the intervention for changing this behaviour was not reported [34]. This is of concern, given that resistance-training is a recommended exercise for breast cancer survivors [10-12], and levels of resistance-training tend to be low among the breast cancer population [64]. There is also evidence that targeting prolonged sedentary behaviour (e.g. sitting) may be an important component of overall daily activity that should also be addressed in behaviour interventions targeted at breast cancer survivors $[9,16]$. One study using an objective measure of physical activity and sitting time showed that reducing sitting time among breast cancer survivors may assist with weight management 
and improve other metabolic outcomes [16]. In addition, a recent systematic review concluded that sedentary behaviour was associated with increased cancer risk for some cancers and cancer mortality among women [9]. To our knowledge, only one study has targeted aerobic and resistance training as well as sedentary behaviour among breast cancer survivors [65]. The results from this study are forthcoming and may provide some useful information. Whilst studies exploring the efficacy of interventions for changing resistance-based activity and sedentary behaviour are needed, whether or not interventions should focus on multiple aspects of a physical activity behaviour (e.g. diet and resistance training), or indeed multiple behaviours is still unclear in the behaviour change field at large [66]. There is a growing recognition that such interventions have a greater potential to impact public health, but there is uncertainty as to whether targeting multiple behaviouurs may be overwhelming for participants [66]. Because of the small number of studies in the current review, it was not possible to examine if efficacy for changing physical activity differed based on whether the intervention was a single or multiple behaviour change intervention. Research examining the relative efficacy of interventions targeting multiple behaviours (e.g. nutrition and physical activity) and multiple aspects of behaviour (e.g. resistance training and aerobic activity and sedentary behaviour ) compared with those targeting single behaviours or single aspects of behaviour are needed to inform future public health practice.

Although this is a comprehensive review of the published literature, there are some limitations that may impact on the generalisability of the findings. Namely, the small number of studies included, the lack of quantitative synthesis, the exclusion of papers not published in English, the methodological weaknesses identified in the included studies (most commonly selection bias) and the possibility of publication bias since grey literature (i.e. unpublished studies) were not examined. However, it should be noted that protocol papers were screened in an attempt to identify grey literature and reduce publication bias, and only one protocol paper fitting eligibility criteria was identified and authors were still at the data collection stage [65]; a qualitative synthesis of the literature was a more appropriate approach under these circumstances than a quantitative approach (i.e. a meta-analysis) because of the heterogeneity and small number of studies conducted in this field [67], and the samples in the included studies reflected the heterogeneity of the breast cancer survivor population. Despite the aforementioned limitations, this review provides insight into the state of the evidence, highlights gaps and limitations in the literature and provides key directions for future research.

In conclusion, although few studies have been conducted examining the efficacy of behavioural interventions for promoting physical activity behaviour change, the majority of studies conducted to date have been of fair methodological quality, have produced changes in physical activity behaviour and may be translatable into sustainable and cost-effective public health approaches. Hence, whilst the field is still in its infancy [3] the results of this review are promising and hope to guide future research.

Acknowledgements CES is supported by a post-doctoral research fellowship from the Institute of Health and Social Science Research, CQ University. RCP is supported by a Senior Research Fellowship from the National Health Medical Research Council of Australia. Infrastructure support was provided by the University of Newcastle, the Hunter Medical Research Institute and CQ University. Claudia Koller and Simon Mierendorff assisted with data extraction.

Conflicts of interest All authors are involved in current trials that are potentially eligible for inclusion in this review.

Open Access This article is distributed under the terms of the Creative Commons Attribution License which permits any use, distribution, and reproduction in any medium, provided the original author(s) and the source are credited.

\section{References}

1. Australian Institute of Health and Welfare and National Breast and Ovarian Cancer Centre, Breast cancer in Australia: an overview, 2009, AIHW: Canberra.

2. Aziz NM. Cancer survivorship research: challenge and opportunity. J Nutr. 2002;132(11):3494S-503.

3. Feuerstein M, Handbook of cancer survivorship. Handbook of cancer survivorship. New York: Springer; 2007. p. 504.

4. Aziz NM, Rowland JH. Trends and advances in cancer survivorship research: challenge and opportunity. Semin Radiat Oncol. 2003;13(3):248-66.

5. Chlebowski R. Lifestyle change including dietary fat reduction and breast cancer outcome. J Nutr. 2007;137(1):233S-5.

6. White $\mathrm{S}$ et al. Translating physical activity interventions for breast cancer survivors into practice: an evaluation of randomized controlled trials. Ann Behav Med. 2009;37(1):10-9.

7. Holick $\mathrm{CN}$ et al. Physical activity and survival after diagnosis of invasive breast cancer. Cancer Epidemiol Biomark Prev. 2008;17(2):379-86.

8. Holmes $\mathrm{M}$ et al. Physical activity and survival after breast cancer diagnosis. JAMA. 2005;25:2479-86.

9. Lynch BM. Sedentary behavior and cancer: a systematic review of the literature and proposed biological mechanisms. Cancer Epidemiol Biomark Prev. 2010;19(11):2691-709.

10. Courneya KS, Mackey JR, McKenzie DC. Exercise for breast cancer survivors: research evidence and clinical guidelines. Physician Sports Med. 2002;30(8):33-42.

11. Hayes $\mathrm{S}$ et al. Australian association for exercise and sport science position stand: optimising cancer outcomes through exercise. J Sci Med Sport. 2009;12:428-34.

12. Schmitz K et al. American College of Sports Medicine roundtable on exercise guidelines for cancer survivors. Med Sci Sports Exerc. 2010;42(7):1409-26.

13. Rock CL, et al. Nutrition and physical activity guidelines for cancer survivors. CA Cancer J Clin. 2012;62:243-74

14. Campbell A, Stevinson C, Crank H. The BASES expert statement on exercise and cancer survivorship. J Sports Sci. 2012;30(9):949-52.

15. Eakin EG et al. Health behaviours of cancer survivors: data from an Australian population-based survey. Cancer Causes Control. 2007;18:881-94. 
16. Lynch BM et al. Objectively measured physical activity and sedentary time of breast cancer survivors, and associations with adiposity: findings from NHANES (2003-2006). Cancer Causes Control. 2010;21(2):283-8

17. Blanchard CM, Courneya KS, Stein K. Cancer survivors' adherence to lifestyle behaviour recommendations and associations with health-related quality of life: results from the American Cancer Society's SCS-II. J Clin Oncol. 2008;26(13):2198-204.

18. Blanchard CM, et al. Current lifestyle behaviours and health-related quality of life in breast, colorectal, and prostate cancer survivors. Research Update. 2003;10(1).

19. Jones LW, Courneya KS. Exercise counseling and programming preferences of cancer survivors. Cancer Pract. 2002;10(4):208-15.

20. Young-McCaughan S, Sexton DL. A retrospective investigation of the relationship between aerobic exercise and quality of life in women with breast cancer. Oncol Nurs Forum. 1991;18(4):751-7.

21. Segar ML et al. The effect of aerobic exercise on self-esteem and depressive and anxiety symptoms among breast cancer survivors. Oncol Nurs Forum. 1998;25(1):107-13.

22. Demark-Wahnefried W et al. Current health behaviours and readiness to pursue life-style changes among men and women diagnosed with early stage prostate and breast carcinomas. Cancer. 2000;88:674-84.

23. Fong DYT et al. Physical activity for cancer survivors: metaanalysis of randomised controlled trials. BMJ. 2012;344:e70.

24. Pollard A, et al. Health behaviour interventions for cancer survivors: an overview of the evidence and contemporary Australian trials. Cancer Forum. 2009;33(3).

25. Michie $\mathrm{S}$ et al. From Theory to Intervention: Mapping Theoretically Derived Behavioural Determinants to Behaviour Change Techniques. Appl Psychol. 2008;57(4):660-80.

26. Bartholomew L, Parcel GS, Kok G. Intervention mapping: a process for developing theory- and evidence-based health education programs. Health Educ Behav. 1998;25(5):545-63.

27. Spark LC et al. Physical activity and/or dietary interventions in breast cancer survivors: a systematic review of the maintenance of outcomes. J Cancer Surviv. 2013;7(1):74-82.

28. Courneya K. Efficacy, effectiveness, and behavior change trials in exercise research. Int J Behav Nutr Phys Act. 2010;7(1):81.

29. Pinto BM, Ciccolo JT. Physical activity motivation and cancer survivorship. Recent Results Cancer Res. 2011;186:367-87.

29. Courneya K, Friedenreich CM. Physical activity and cancer control. Semin Oncol Nurs. 2007;23(4):242-52.

30. Michie $\mathrm{S}$ et al. A refined taxonomy of behaviour change techniques to help people change their physical activity and healthy eating behaviours: the CALO-RE taxonomy. Psychol Heal. 2011;26(11):1479-98.

31. Zaza $S$ et al. Data collection instrument and procedure for systematic reviews in the Guide to Community Preventive Services. Task Force on Community Preventive Services. Am J Prev Med. 2000;18(1 Suppl):44-74.

32. Thomas $\mathrm{BH}$ et al. A process for systematically reviewing the literature: providing the research evidence for public health nursing interventions. Worldviews Evid Based Nurs. 2004;1(3):176-84.

33. Basen-Engquist $\mathrm{K}$ et al. Randomized pilot test of a lifestyle physical activity intervention for breast cancer survivors. Patient Educ Couns. 2006;64(1-3):225-34.

34. Bloom JR et al. Addressing the needs of young breast cancer survivors at the 5 year milestone: can a short-term, low intensity intervention produce change? J Cancer Surviv. 2008;2(3):190-204.

35. Daley AJ et al. Randomized trial of exercise therapy in women treated for breast cancer. J Clin Oncol. 2007;25(13):1713-21.

36. Kim $\mathrm{SH}$ et al. Randomized pilot test of a simultaneous stagematched exercise and diet intervention for breast cancer survivors. Oncol Nurs Forum. 2011;38(2):E97-106

37. Matthews CE et al. Evaluation of a 12-week home-based walking intervention for breast cancer survivors. Support Care Cancer. 2007;15(2):203-11.
38. Pakiz B et al. Effects of a weight loss intervention on body mass, fitness, and inflammatory biomarkers in overweight or obese breast cancer survivors. Int J Behav Med. 2011;18(4):333-41.

39. Pinto BM et al. Home-based physical activity intervention for breast cancer patients. J Clin Oncol. 2005;23(15):3577-87.

40. Rogers LQ et al. A randomized trial to increase physical activity in breast cancer survivors. Med Sci Sports Exerc. 2009;41(4):93546.

41. Vallance JKH et al. Randomized controlled trial of the effects of print materials and step pedometers on physical activity and quality of life in breast cancer survivors. J Clin Oncol. 2007;25(17):23529.

42. Pinto BM et al. Maintenance of effects of a home-based physical activity program among breast cancer survivors. Support Care Cancer. 2008;16(11):1279-89.

43. Rogers LQ et al. Physical activity and health outcomes three months after completing a physical activity behavior change intervention: persistent and delayed effects. Cancer Epidemiol Biomarkers Prev. 2009;18(5):1410-8.

44. Vallance JK et al. Maintenance of physical activity in breast cancer survivors after a randomized trial. Med Sci Sports Exerc. 2008;40(1):17380.

45. Vallance JK et al. Development and evaluation of a theory-based physical activity guidebook for breast cancer survivors. Health Educ Behav. 2008;35(2):174-89.

46. Daley AJ et al. Exercise therapy in women who have had breast cancer: design of the Sheffield women's exercise and well-being project. Heal Educ Res. 2004;19(6):686-97.

47. Rabin C, BM Pinto, and G. Frierson Mediators of a randomized controlled physical activity intervention for breast cancer survivors. J Sport Exerc Psychol. 2006; 269-284.

48. Rogers LQ et al. Reduced barriers mediated physical activity maintenance among breast cancer survivors. J Sport Exerc Psychol. 2011;33(2):235-54.

49. Vallance $J$ et al. Understanding physical activity maintenance in breast cancer survivors. Am J Heal Behav. 2010;34(2):22536.

50. Hatchett A, JS Hallam, and Ford MA. Evaluation of a social cognitive theory-based email intervention designed to influence the physical activity of survivors of breast cancer. Psychooncol. 2012;10(10).

51. Prochaska JO, Velicer WF. The transtheoretical model of health behavior change. Am J Heal Promot. 1997;12(1):38-48.

52. Bandura A. Social Foundations of thought and action: a social cognitive theory. New York: Prentice Hall, 1986.

53. Ajzen I. The theory of planned behavior. Organ Behav Hum Decis Process. 1991;50:179-211.

54. Vallance JKH et al. Analyzing Theoretical Mechanisms of Physical Activity Behavior Change in Breast Cancer Survivors: Results from the Activity Promotion (ACTION) Trial. Ann Behav Med. 2008;35(2):150-8.

55. Glasgow RE et al. The RE-AIM framework for evaluating interventions: what can it tell us about approaches to chronic illness management? Patient Educ Couns. 2001;44(2):119-27.

56. Glasgow RE, Emmons KM. How can we increase translation of research into practice? Types of evidence needed. Annu Rev Public Health. 2007;28:413-33.

57. Glasgow RE, Vogt TM, Boles SM. Evaluating the public health impact of health promotion interventions: the RE-AIM framework. Am J Public Health. 1999;89:1322-7.

58. Michie S, Abraham C. Interventions to change health behaviours: evidence-based or evidence-inspired? Psychol Heal. 2004;19:2949.

59. Glanz K, Bishop DB. The role of behavioral science theory in development and implementation of public health interventions. Annu Rev Public Health. 2010;31:399-418. 
60. Noar S, Benac C, Harris M. Does tailoring matter? Meta-analytic review of tailored print health behavior change interventions. Psychol Bull. 2007;133(4):673-93.

61. Vandelanotte $\mathrm{C}$ et al. Website-delivered physical activity interventions: a review of the literature. Am J Prev Med. 2007;33(1):54-64.

62. Short $\mathrm{C}$ et al. Efficacy of tailored-print interventions to promote physical activity: A systematic review of randomised trials. Int J Behav Nutr Phys Act. 2011;8(1):113.

63. Kuijpers $W$ et al. A systematic review of web-based interventions for patient empowerment and physical activity in chronic diseases: relevance for cancer survivors. J Med Internet Res. 2013;15(2):e37.
64. Harrison S, Hayes SC, Newman B. Level of physical activity and characteristics associated with change following breast cancer diagnosis and treatment. Psychooncology. 2009;18(4):387-94.

65. Short $\mathrm{C}$ et al. Move more for Life: the protocol for a randomised efficacy trial of a tailored-print physical activity intervention for posttreatment breast cancer survivors. BMC Cancer. 2012;12(1):172.

66. Prochaska JJ, Prochaska JO. A review of multiple health behavior change interventions for primary prevention. Am J Lifestyle Med. 2011;5(3):208-21.

67. Rychetnik L et al. A glossary for evidence based public health. J Epidemiol Community Health. 2004;58:538-45. 